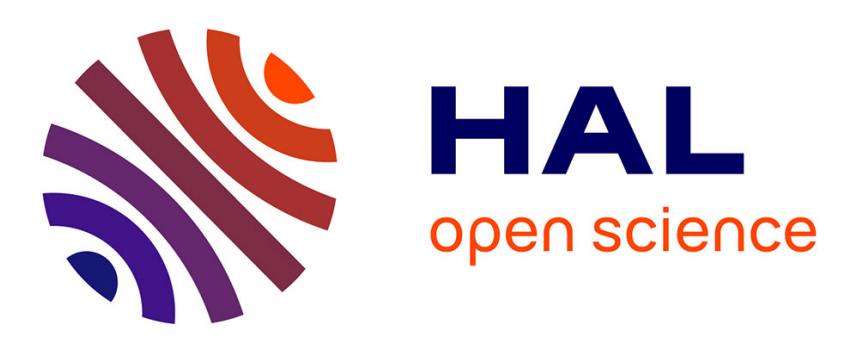

\title{
Insight into electrocaloric cooling power in multilayer capacitors using infra-red camera
}

\author{
Yang Liu, Hervé Strozyk, Brahim Dkhil, Emmanuel Defay
}

\section{To cite this version:}

Yang Liu, Hervé Strozyk, Brahim Dkhil, Emmanuel Defay. Insight into electrocaloric cooling power in multilayer capacitors using infra-red camera. Applied Physics Letters, 2016, 109, pp.212902. 10.1063/1.4968581. hal-01782592

\section{HAL Id: hal-01782592 \\ https://hal.science/hal-01782592}

Submitted on 24 Aug 2020

HAL is a multi-disciplinary open access archive for the deposit and dissemination of scientific research documents, whether they are published or not. The documents may come from teaching and research institutions in France or abroad, or from public or private research centers.
L'archive ouverte pluridisciplinaire HAL, est destinée au dépôt et à la diffusion de documents scientifiques de niveau recherche, publiés ou non, émanant des établissements d'enseignement et de recherche français ou étrangers, des laboratoires publics ou privés. 


\section{Insight into electrocaloric cooling power in multilayer capacitors using infra-red camera}

Cite as: Appl. Phys. Lett. 109, 212902 (2016); https://doi.org/10.1063/1.4968581

Submitted: 11 August 2016 . Accepted: 11 November 2016 . Published Online: 22 November 2016

Yang Liu, Hervé Strozyk (D), Brahim Dkhil, and Emmanuel Defay
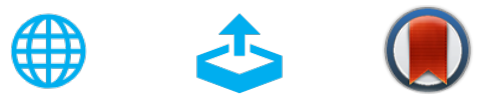

View Online

Export Citation

\section{ARTICLES YOU MAY BE INTERESTED IN}

Direct and indirect measurements on electrocaloric effect: Recent developments and perspectives

Applied Physics Reviews 3, 031102 (2016); https://doi.org/10.1063/1.4958327

Direct electrocaloric measurements of a multilayer capacitor using scanning thermal microscopy and infra-red imaging

Applied Physics Letters 102, 032903 (2013); https://doi.org/10.1063/1.4788924

An electrocaloric refrigerator with direct solid to solid regeneration

Applied Physics Letters 110, 243503 (2017); https://doi.org/10.1063/1.4986508

\section{Lock-in Amplifiers up to $600 \mathrm{MHz}$}
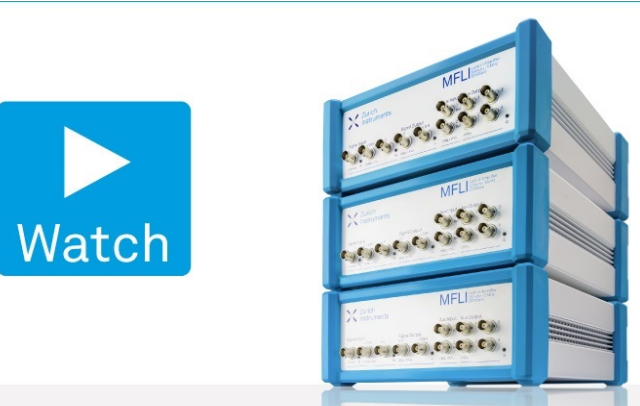


\title{
Insight into electrocaloric cooling power in multilayer capacitors using infra-red camera
}

\author{
Yang Liu, ${ }^{1,2, a)}$ Hervé Strozyk, ${ }^{2}$ Brahim Dkhil, ${ }^{1}$ and Emmanuel Defay ${ }^{2, a)}$ \\ ${ }^{1}$ Laboratoire Structures, Propriétés et Modélisation des Solides (SPMS), CentraleSupélec, CNRS UMR8580, \\ Université Paris-Saclay, 92290 Châtenay-Malabry, France \\ ${ }^{2}$ Materials Research and Technology Department, Luxembourg Institute of Science and Technology (LIST), \\ 41 Rue du Brill, L-4422 Belvaux, Luxembourg
}

(Received 11 August 2016; accepted 11 November 2016; published online 22 November 2016)

\begin{abstract}
Compact multilayer capacitors (MLCs) have attracted strong interest as the most promising elements for the design of electrocaloric prototypes. Recent theoretical simulations have predicted that MLCs could permit a sustained cooling power. However, direct experimental evidence is still lacking. Here, we use an infra-red camera to characterize the cooling power of commercial MLCs by combining both spatially and temporally resolved measurements. We also compare the experimental data with theoretical models in order to highlight the routes for developing and optimizing the future MLC-based devices as well as the measurement conditions and modeling tools. Published by AIP Publishing.

[http://dx.doi.org/10.1063/1.4968581]
\end{abstract}

There has been a recent upsurge in research on the electrocaloric (EC) effect to seek for alternative solutions to replace the current vapor-compression cooling devices. ${ }^{1-6}$ The EC effect refers to an isothermal entropy change or an adiabatic temperature change when an EC material is subjected to a perturbation in the external electric field. After almost 10 years of research on EC materials initialing from the discovery of a giant EC effect in antiferroelectric thin films, ${ }^{1}$ the current interest focuses on direct measurements and the design of prototypes while this cooling technology remains in its infancy compared to its magnetocaloric counterpart. ${ }^{6}$ Recently, several thermodynamic EC refrigerations (see one example of Brayton-like type in Fig. 1) for cooling applications were theoretically proposed and conceptually demonstrated especially using multilayer capacitors (MLCs). ${ }^{7-11}$ MLCs make a compromise between thin films with giant EC responses and bulk with larger active volumes, which are considered as the most promising configurations for developing EC cooling devices. ${ }^{3,5,6}$

Although the EC effect in MLCs has been known for almost 30 years, ${ }^{12,13}$ it is only recently that the role of metallic inner electrodes in conducting EC heat has been addressed. ${ }^{14,15}$ Moreover, depending on EC materials and electrodes, a sustained cooling power of $36 \mathrm{~kW} / \mathrm{m}^{2}$ was predicted by using a lumped thermal model. ${ }^{15}$ Later, a value of $81.7 \mathrm{~kW} / \mathrm{m}^{2}$ was refined by using finite element modelling. ${ }^{16}$ Interestingly, these values may compete favorably with the most advanced magnetocaloric refrigerators based on Gd for which $\sim 2.5 \mathrm{~kW} / \mathrm{m}^{2}$ is a typical value of cooling power. ${ }^{17}$ However, there is no experimental evidence directly measuring this important parameter of EC devices to inspire confidence for practical applications and guide researchers towards EC prototypes with enhanced performances. $^{7-16,18,19}$ In this work, we carried out IR measurements on standard operating MLCs, which gave the opportunity to extract its cooling power that we used to reveal the

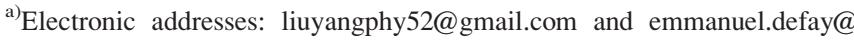
list.lu
}

parameters to play with in order to optimize MLC-based prototypes and develop more accurate modeling tools and careful measurement conditions.

Before showing the experimental results of the cooling power of MLCs, let us first focus on the basic cooling mechanism taking into account the geometrical configurations of MLCs (i.e., electroactive multilayers interconnected with inner electrodes in contact with external metallic terminals). A Brayton-like EC refrigeration cycle $e^{3,6,8}$ using MLCs can be described as follows: (1-2) adiabatic application of the external electric field (polarizing); (2-3) isofield cooling of the MLCs accompanied by heat exchange with hot sink; (3-4) adiabatic removal of external stimulus (depolarizing); and (4-1) isofield heating of the MLCs associated with the heat exchange with cold load. In this refrigeration cycle, the metallic terminals are of great importance to extract the EC heat flux in steps (2-3) and (4-1). ${ }^{20}$ Interestingly, our recent experimental study allowed us to directly deduce the EC heat flux crossing MLCs by using an infra-red camera, and it

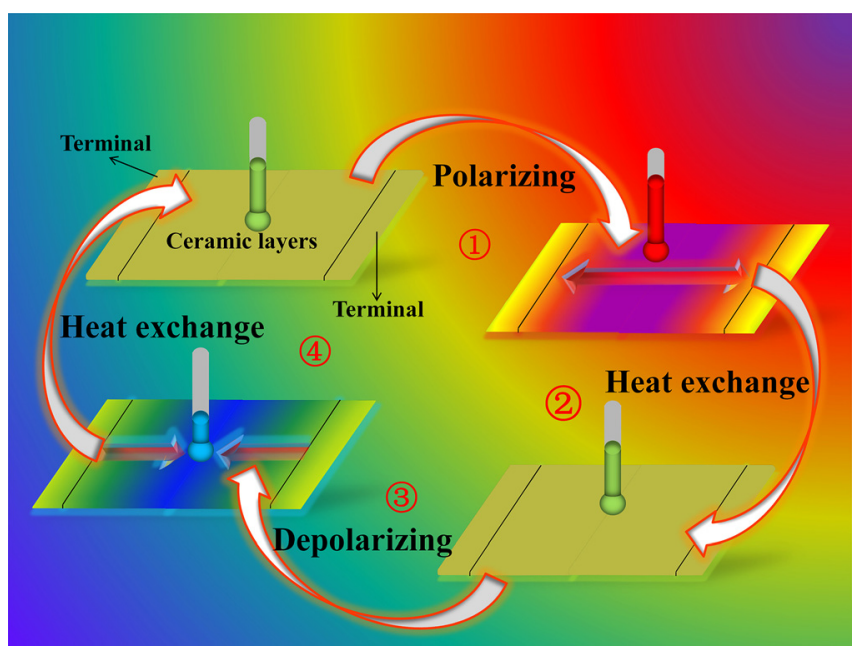

FIG. 1. A sketch of Brayton-like EC refrigeration cycle using MLCs. The horizontal red arrows indicate the main direction of EC heat flux. 
(a)
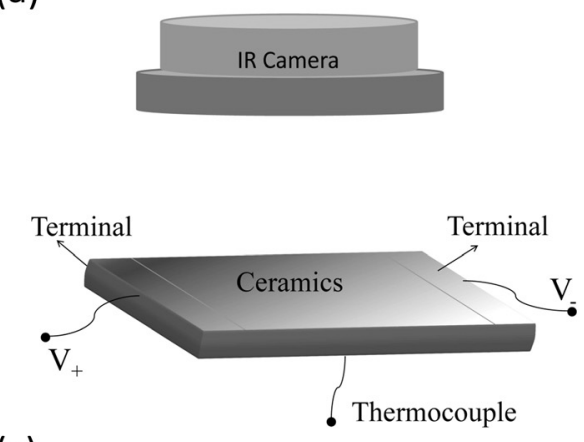

(c)

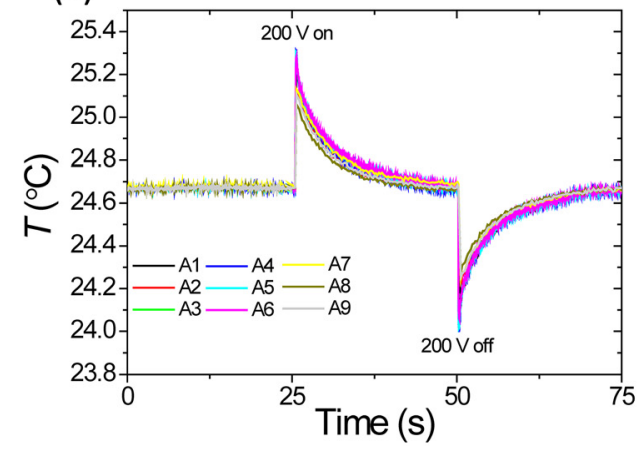

(b)

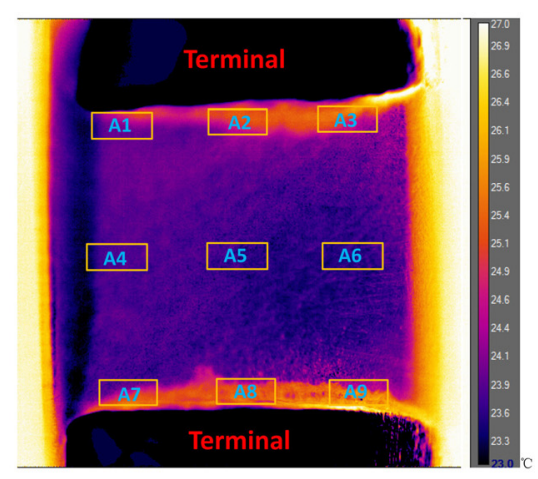

(d)

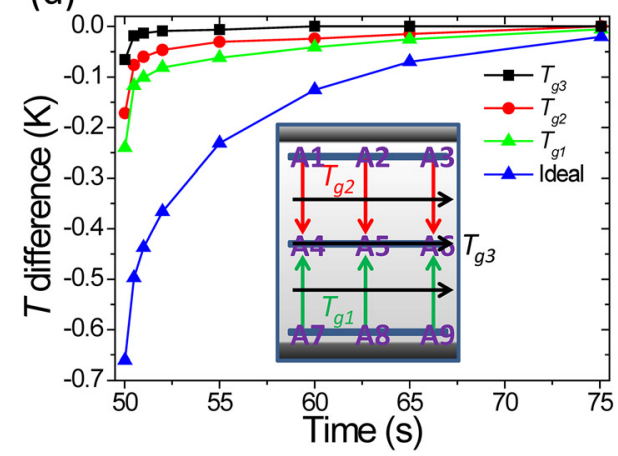

FIG. 2. (a) The schematic representation of the measurement setup. (b) The typical thermal image of the top surface of one MLC at room temperature without applied voltage, on which the regions numbered from A1 to A9 $(\sim 520 \mu \mathrm{m}$ $\times \sim 200 \mu \mathrm{m}$ ) have been reported. (c) Recorded temperatures of the different regions of (b) versus time when $200 \mathrm{~V}$ have been applied or removed. (d) Temperature difference between the different selected regions is extracted from (c). The inset depicts the corresponding locations of the selected regions and the temperature difference. Arrows indicate the directions of heat flow. was found that the heat flux parallel with the terminalterminal direction is three times larger than the perpendicular one. ${ }^{20}$ As a result, Fig. 1 only shows schematically the main direction of EC heat flow through arrows between the central ceramic regions and the two terminals. Although Braytonlike EC cycles have been employed in previous works, ${ }^{3,6,8}$ the heat transfer as shown in Fig. 1 is not explicitly indicated, which is of great importance for design of suitable EC devices.

As illustrated in Fig. 2(a), in this study, we use a FLIR X6580SC camera (Thermal resolution: $20 \mathrm{mK}$; spatial resolution of $6.5 \mu \mathrm{m}$ ), and MLCs are purchased from Multicomp (MC1210F476Z6R3CT). The MLCs are made of around 150 doped $\mathrm{BaTiO}_{3}$ ceramic layers with the thickness of $11 \mu \mathrm{m}$ separated by interdigitated $\mathrm{Ni}$ inner electrodes with the thickness of $2.0 \mu \mathrm{m}$. We deposit tin onto the terminals to realize electrical connection by thin copper wires between the MLCs and voltage generator (KEITHLEY). 800-grit and 1200-grit abrasive papers are sequentially used to polish the top surface of MLCs. After that, ethanol is employed to wash and clean the MLCs. The IR camera has been calibrated with a black body on the same temperature range as these experiments by the manufacturer (FLIR). Then, all experiments presented in this paper were performed after having reached temperature stability of the whole set-up. Consequently, the temperature was stabilized on the MLC prior to any voltage supply. This temperature was measured with a Pt-100 thermometer (TE Connectivity Ref NB-PTCO155 class $F$ 0.1) with the accuracy of $\pm 100 \mathrm{mK}$, regarding the absolute temperature. The precision of variation of temperature is much better as the temperature coefficient of resistance is $3850 \mathrm{ppm} /{ }^{\circ} \mathrm{C}$ and our electronic reading system can reach the ppm, which gives approximately $1 \mathrm{mK}$ as the most sensitive temperature variation we can extract from the Pt-100 thermometer. Similar experimental set-up can be also found in Refs. 20 and 21.
Fig. 2(b) shows the zero-voltage thermal image of the top surface of one MLC at room temperature. It is observed that factitious temperature spatial variations only exist near the interface between the metallic terminals and ceramic layers. It is due to the surface emissivity variations on the MLCs due to the difference in materials (high emissivity ceramic and low emissivity metallic terminals). ${ }^{21}$ As already mentioned in our previous work, ${ }^{20}$ we adjusted the emissivity of each point of the MLC surface in order to obtain the same temperature on the whole MLC surface prior to applying any voltage to the MLC (see Table I). This operation can be done with the camera software (IResearch) once the temperature has been set with thermocouple [Fig. 2(a)]. Local variations appear on the surface of the MLC, especially close to the terminals (see Table I). It can even occur that emissivity reaches 1 . Note that this happens exclusively in regions A1-A3 and A7-A9 [Fig. 2(b)] located extremely close to the terminal regions but not on the terminals themselves. As the terminals are made of metal, their emissivity is low (less than 10\%), and therefore, their reflectivity is very intense. This high reflection infers an extrinsic increase of the

TABLE I. Emissivity of various regions of the top surface of MLCs before the EC effect is activated.

\begin{tabular}{lc}
\hline \hline Region & Emissivity \\
\hline A1 & 0.788 \\
A2 & 0.947 \\
A3 & 0.940 \\
A4 & 0.839 \\
A5 & 0.750 \\
A6 & 0.743 \\
A7 & 0.880 \\
A8 & 0.980 \\
A9 & 1.000 \\
\hline \hline
\end{tabular}


effective emissivity of the ceramic regions close to the terminals, even when polishing has been performed. For instance, the average emissivity of A4-A6 areas is 0.777 whereas it is as high as 0.923 for $\mathrm{A} 1-\mathrm{A} 3$ and $\mathrm{A} 7-\mathrm{A} 9$ regions. Thanks to this emissivity adjustment, the final temperatures extracted are much more accurate as IR cameras only detect photons. This requires that the emissivity of the measured regions is known. It is, however, true that reaching saturation with an effective emissivity of 1 induces a slight error on the temperature extraction, estimated to be less than $2 \%$, based on the data in Table I.

Fig. 2(c) shows the typical results for Brayton-like EC refrigeration cycles where the surroundings play the role of both load and sink. A significant distribution in the recorded temperature profile is evidenced, which is consistent with our previous work. ${ }^{20}$ Combining both spatially and temporally resolved measurements, Fig. 2(d) summarizes the extracted temperature difference between different selected regions when a voltage of $200 \mathrm{~V}$ has been removed. Specifically, $T_{g 1}$ and $T_{g 2}$ indicate the difference in temperature between the average temperature corresponding to (A4, $\mathrm{A} 5$, and A6) regions and the one averaged, respectively, over the three regions (A7, A8, and $\mathrm{A} 9)$ and $(\mathrm{A} 1, \mathrm{~A} 2$, and $\mathrm{A} 3)$. $T_{g 3}$ stands for the average difference between (A1, A4, and A7) and (A3, A6, and A9) regions. The ideal temperature difference refers to the one expected between central regions (A4, A5, and A6) and EC inactive terminals (the temperature is constant while operating). ${ }^{14}$ It is shown that the magnitude of $T_{g \mathrm{i}}(\mathrm{i}=1,2,3)$ experiences a dramatic decrease during the first $2 \mathrm{~s}$. Moreover, the thermal time constant $\tau$ defined as the time that it takes for the MLC to reach $63.2 \%$ of its steadystate temperature value with respect to its initial value is about $1 \mathrm{~s}$. The difference between $T_{g 1}$ and $T_{g 2}$ arises from the different thermal masses due to the different amount of $\mathrm{SnPb}$ deposited onto the terminals in order to ensure electrical connections. ${ }^{20}$

Now, let us calculate the cooling power of MLCs using a lumped thermal model, which is available in the field of electrocaloric literature. ${ }^{14}$ In a lumped equivalent thermal circuit, taking into account the heat capacity and the thermal conductivity of $\mathrm{BaTiO}_{3}$ and $\mathrm{Ni}$ electrodes, the temporal cooling capacity $\dot{Q}$ follows an exponential decrease $\dot{Q}=\dot{Q}_{\text {max }}$ $\exp \left(-t / R_{\text {total }} C_{\text {total }}\right) .{ }^{14} \dot{Q}_{\max }=T_{\mathrm{i}} / R_{\text {total }}$ is the initial EC cooling capacity at $t=0 \mathrm{~s}$. $R_{\text {total }}=R_{\mathrm{TH}}^{\mathrm{Ni}} / n$ is the total thermal resistance in series, where $R_{\mathrm{TH}}^{\mathrm{Ni}}=L / 2 / \kappa_{\mathrm{Ni}} A_{\mathrm{Ni}}$ is the thermal resistance of one single $\mathrm{Ni}$ electrode ( $L$ : effective length, $A_{\mathrm{Ni}}$ : cross-sectional area) and $n$ is the total number of $\mathrm{Ni}$ electrodes (150 in our case). $C_{\text {total }}$ is the total thermal capacitance where $C_{\text {total }}=n\left(C_{\mathrm{TH}}^{\mathrm{Ni}}+2 C_{\mathrm{TH}}^{\mathrm{EC}}\right) . C_{\mathrm{TH}}^{\mathrm{Ni}}=66 \mu \mathrm{J} / \mathrm{K}$ (Ref. 14) and $C_{\mathrm{TH}}^{\mathrm{EC}}=\rho_{\mathrm{EC}} c_{\mathrm{EC}}\left(v_{\mathrm{EC}} / 2\right)=111.5 \mu \mathrm{J} / \mathrm{K}$ are the thermal capacitance of $\mathrm{Ni}$ and $\mathrm{EC}$ layer $\left(\mathrm{BaTiO}_{3}\right)$, respectively, where $\rho_{\mathrm{EC}}=5840 \mathrm{~kg} / \mathrm{m}^{3}$ and $v_{\mathrm{EC}}=8.8 \times 10^{-11} \mathrm{~m}^{3}$ are the mass density and volume of $\mathrm{BaTiO}_{3}$ layer, respectively. Note that the expression of $C_{\text {total }}$ used here differs from the previous work. ${ }^{14}$ We specifically consider $C_{\text {total }}$ as a sum of the thermal masses of different components. In this regard, $C_{\text {total }}$ is enhanced by a factor of about 5 compared to that obtained in the previous result. ${ }^{14}$ This may lead to a significant change in the magnitude of heat flux and cooling power.
Now, we may calculate the thermal relaxation time $\tau=R_{\text {total }} C_{\text {total }}$. Specifically, $C_{\text {total }}$ is calculated as $43350 \mu \mathrm{J} / \mathrm{K}$ while $R_{\text {total }}=22.7 \mathrm{~K} / \mathrm{W}$, and the thermal relaxation time $\tau$ is about $0.984 \mathrm{~s}$, which is well consistent with our measured value of $1 \mathrm{~s}$ [see Fig. 2(d)]. Moreover, we can calculate the initial $Q_{\max }\left(Q_{\max }=T_{\mathrm{i}} / R_{\text {total }}\right)$ as $10.60 \mathrm{~mW}, 7.56 \mathrm{~mW}$, and $2.86 \mathrm{~mW}$ by using $R_{\text {total }}=22.7 \mathrm{~K} / \mathrm{W}$ and $T_{\mathrm{i}}=0.240 \mathrm{~K}$, $0.172 \mathrm{~K}$, and $0.0655 \mathrm{~K}$ for heat flux 1,2 , and 3 experimentally extracted from Fig. 2(d). A large cooling power takes place during the first $5 \mathrm{~s}$, and it decays significantly after removing the external voltage, as shown in Fig. 3(a). The largest $\dot{Q}_{\max }$ for heat flow 1 is about two times smaller than the limiting case $(28.86 \mathrm{~mW})$ for which $T_{\mathrm{i}}$ is set in order to obtain the ideal temperature difference between the central regions and terminals extracted from Fig. 2(d). The average cooling capacity $Q_{\mathrm{av}}=\dot{Q}_{\max } / 20$ is $0.53 \mathrm{~mW}, 0.38 \mathrm{~mW}$, and $0.14 \mathrm{~mW}$ for heat fluxes 1,2 , and 3 . As a result, the cooling powers of heat fluxes 1,2 , and 3 , thus, correspond to $0.21 \mathrm{~kW} / \mathrm{m}^{2}, 0.15 \mathrm{~kW} / \mathrm{m}^{2}$, and $0.048 \mathrm{~kW} / \mathrm{m}^{2}$, respectively. These values including the limiting case $\left(0.57 \mathrm{~kW} / \mathrm{m}^{2}\right)$ are still smaller than the recent theoretical predictions of $\sim 0.8-2.5 \mathrm{~kW} / \mathrm{m}^{2}$ in $\mathrm{BaTiO}_{3}$-based MLCs with $\mathrm{Ni}$ electro$\operatorname{des}^{16}$ and that $\left(\sim 2.5 \mathrm{~kW} / \mathrm{m}^{2}\right)$ in magnetocaloric refrigerators based on Gd. ${ }^{17}$ It was reported that the cooling power of MLCs is limited by the thermal relaxation time. ${ }^{16}$ Our
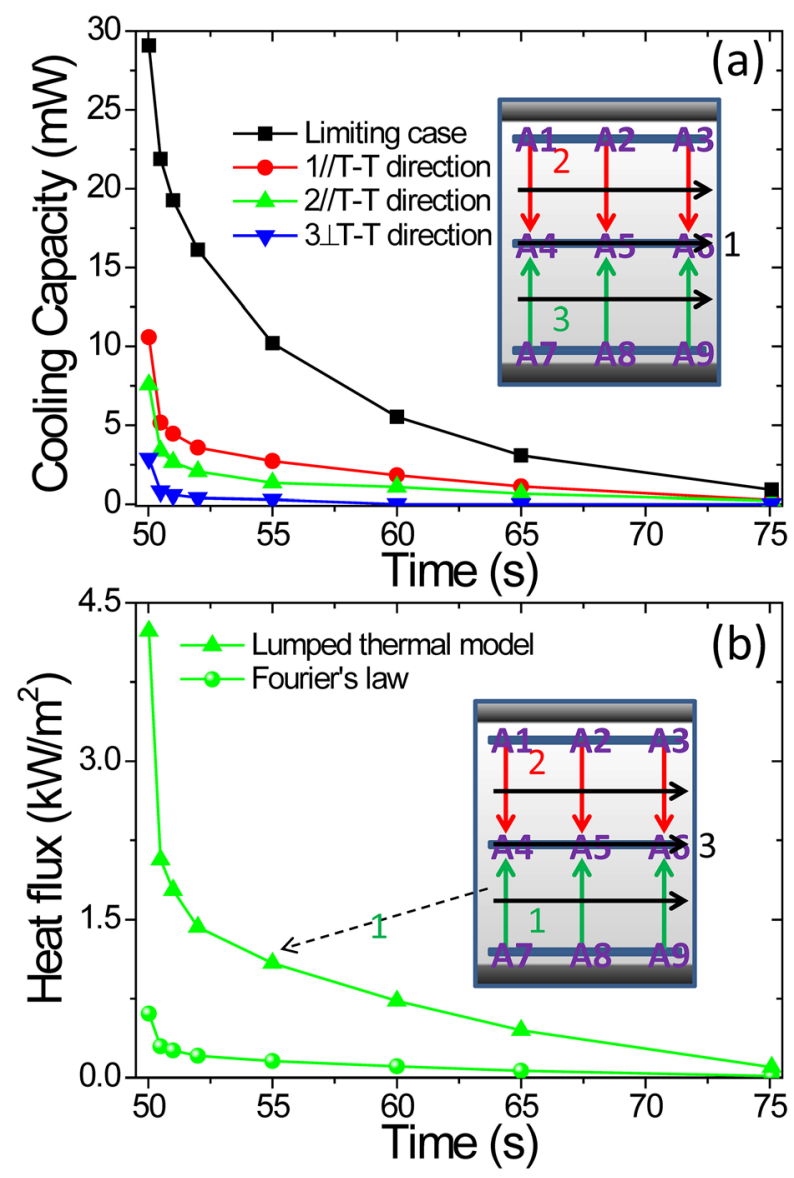

FIG. 3. (a) EC cooling capacity associated with heat fluxes 1,2 , and 3 as a function of real time when $200 \mathrm{~V}$ is removed. The limiting case corresponds to the idea temperature gradient as shown in Fig. 2(d). (b) Comparison of calculation results on the heat flux 1 between a lumped thermal model and Fourier's law as a function of real time when $200 \mathrm{~V}$ is removed. 
findings here indicate that very fast heat exchange cannot be simply realized using our current geometry of devices (our measured $\tau$ is larger than theoretical expectations by finite element modeling ${ }^{16}$ ), which may therefore prevent reaching a larger cooling power. As a result, a strong evacuation of the heat imposed on the terminals and/or further geometric optimization $^{16}$ and/or good thermal transfer adhesive attached to MLCs are very desired in future works.

The lumped thermal model itself may also overestimate the thermal relaxation time compared to that obtained by finite element modeling. ${ }^{16}$ As a result, smaller cooling power may be predicted compared to the results deduced from the finite element analysis. ${ }^{16}$ In addition to finite element modeling, other direct theoretical studies are highly desirable to understand heat distribution and transfer in MLCs. For instance, by solving the thermal equations, one can describe the side flow and a variety of conditions on the MLC borders. ${ }^{9}$ In order to evaluate the cooling power results [(Fig. 3(a)] obtained through the lumped thermal model, we also make further estimations based on Fourier's law $\dot{Q}_{\mathrm{h}}=-\kappa_{\mathrm{eff}} T_{\mathrm{i}} / l$, where $\dot{Q}_{\mathrm{h}}$ is the rate of heat flux, $\kappa_{\text {eff }}$ is the effective thermal conductivity combining contributions from ceramic layers and inner electrodes, $l$ is the heat transfer length, and $T_{\mathrm{i}}$ is the average temperature difference obtained from Fig. 2(d). The comparison of the results between the lumped thermal model and Fourier's law for heat flux 1 is shown in Fig. 3(b). It is found that the average cooling power for heat flux 1 is $0.030 \mathrm{~kW} / \mathrm{m}^{2}$, which is 6 times smaller than the one obtained from the lumped thermal model $\left(0.21 \mathrm{~kW} / \mathrm{m}^{2}\right)$. Therefore, our results show that considering the thermal conductivity of MLCs merely as a geometric average of the thermal conductivity of $\mathrm{BaTiO}_{3}$ and $\mathrm{Ni}$ may lead to inaccurate estimations of heat flow and cooling power.

Our previous EC results were obtained under different voltage sequences from $25 \mathrm{~V}$ to $200 \mathrm{~V},{ }^{20}$ which is accompanied with a temperature change before running the EC experiments except for the very beginning. Here, we waited for about $25 \mathrm{~s}$ before the EC effect was triggered as shown in Fig. 2(c). Interestingly, no appreciable discrepancies in the temperature change and thermal time constant have been spotted between these two results, which rule out the nonequilibrium contributions due to the temperature variation in our previous work. ${ }^{20} \mathrm{We}$ also note that the slight change in the thermal relaxation time under different voltage as implied in our previous work ${ }^{20}$ cannot be well understood by the lumped thermal model, which assumes that MLCs are perfectly thermally isolated from the surroundings. ${ }^{14}$ This is not exactly the case in our experimental measurement without any thermal shield. In this case, the heat exchange between the MLC and the surroundings also make contributions especially when the voltage is not high enough. This is also the reason why we only present the heat flux under $200 \mathrm{~V}$ rather than in the low voltage regime. Under high voltages, the system can be safely regarded to be in nearly adiabatic conditions.

Finally, we also carried out a thermal simulation of the electrocaloric device based on MLCs to show the role of the heat transfer with its environment and stress possible origins of errors one can make during measurements. The equivalent electrical circuit employed in this model is shown in Fig. 4(a). Basically, the thermal model takes into account the thermal resistance of the MLC edge and electrical supply plate $R_{\text {ce }}$, heat transfer in a dielectric fluid via forced convection $R_{\mathrm{ef}}$, and heat transfer to the housing of the active elements $R_{\text {out }}$. It also takes into account the thermal capacitances of the MLCs $C_{\mathrm{c}}$ and of the fluid $C_{\mathrm{f}}$. $Q_{\text {in }}$ stands for the heat created by the EC effect into the MLC.

The thermal behavior of one MLC in contact with a fluid is shown in Fig. 4(b). It can be seen that the temperature of the fluid $\theta_{\mathrm{f}}$ is not decreasing as low as that of the MLC $\theta_{\mathrm{c}}$. For information, Fig. 4(b) also displays how EC heat is temporally given to the system. Some clues about this behavior can be given. First, one can introduce equivalent thermal mass to compare temperature evolutions of both MLC and

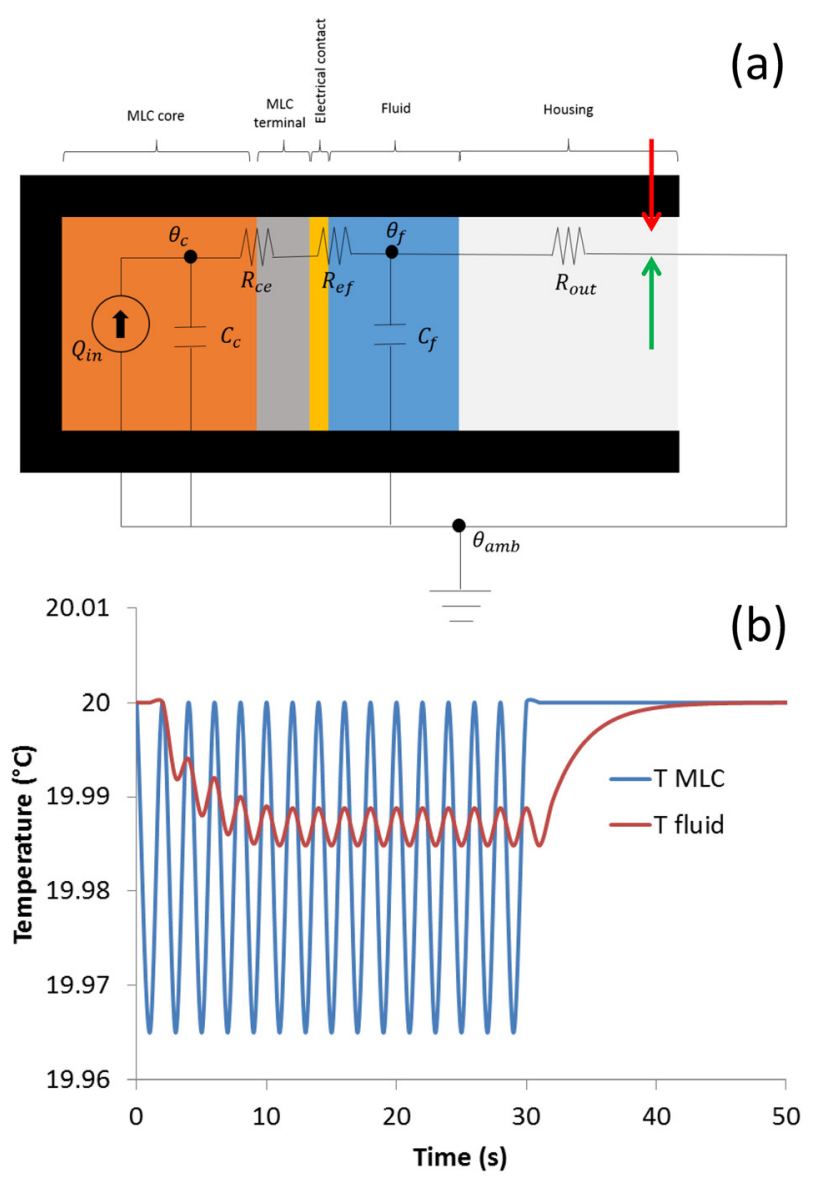

FIG. 4. (a) Physical model of the MLC in contact with a convective fluid canal. Heat is exchanged from the MLC core to the environment via MLC metallic terminal, external electrical contact layer, fluid, and eventually containment housing. The equivalent electrical/thermal circuit is also represented. Heat created by the MLC is represented as a heat source $Q_{i n}$. This heat is then sent to the metallic terminal of the MLC via $R_{c e}$ and then to the fluid via $R_{e f}$. The fluid finally releases the heat to the environment via $R_{\text {out }}$. Heat exchanges are also modulated by the thermal capacities $C_{c}$ and $C_{f}$. This models the ability of, respectively, the MLC and the fluid to store momentously the heat. The system is monitored by $\theta_{c}, \theta_{f}$, and $\theta_{a m b}$, which are, respectively, the MLC, the fluid, and environment temperatures. Environment is represented as the thermal sink of the system. (b) Temporal evolution of $\theta_{c}$ (red curve) and $\theta_{f}$ (blue curve). Electrocaloric heat pulses are generated, and the temperature response of the fluid is monitored. Temperature variation of the fluid is smaller than that of the MLC. The fluid first starts to heat up, before it reaches a temperature plateau when a new thermal periodic steady state is reached. When MLC stops injecting heat in the system, the temperature of the fluid then falls down exponentially to equilibrium with environment temperature. 
fluid. The heat generated by the MLC can be expressed as $Q=\rho V C_{p} \Delta T$. In our model, the equivalent thermal mass $m_{T}=\rho V C_{p}$ of the MLC is twice smaller than that of the fluid. So, for a given heat pulse provided by the MLC, if we assume that all this heat is transferred to the fluid, the fluid undergoes a smaller $\Delta T$ than that of MLC. However, thermal equilibrium is a trade-off between heat income, storage, and release to the environment. This last heat transfer is important to try to explain the difference between the models and the measurements. Because the surroundings of the MLC are not controlled (atmosphere, thermal conductive contacts, radiation thermal exchanges, etc.), major differences can be obtained. Further optimizations of this model are highly desired to understand the regenerative effect using MLCs. Nevertheless, combining this simplistic model with our experimental data in this work may provide a practical solution.

In summary, we have demonstrated the useful role of an IR camera in measuring the cooling power of MLCs, which provides a practical route to characterize and select proper MLCs for designing suitable devices. Moreover, our study combines theoretical simulations and experimental measurements to achieve optimizations of performances such as geometrical adjustments. We hope that our results may inspire more works on developing EC prototypes for refrigeration applications as well as improved measurement procedures and more accurate modeling tools.

Y. L. and B. D. acknowledge a public grant overseen by the French National Research Agency (ANR) as part of the "Investissements d'Avenir" programme (reference: ANR10-LABX-0035, Labex NanoSaclay). E. D. acknowledges Fonds National de la Recherche from Luxembourg for the support through COFERMAT PEARL project (FNR/P12/ 4853155). E. D. and B. D. acknowledge Fonds National de la Recherche (FNR) du Luxembourg through the InterMobility project 16/1159210 "MULTICALOR". The authors are grateful to R. Faye and D. Sette for fruitful discussions. The authors gratefully thank the anonymous reviewer for his/her suggestions about the thermal mass of MLCs in lumped thermal model to improve the manuscript.

\footnotetext{
${ }^{1}$ A. S. Mischenko, Q. Zhang, J. F. Scott, R. W. Whatmore, and N. D. Mathur, Science 311, 1270 (2006).

${ }^{2}$ B. Neese, B. Chu, S. G. Lu, Y. Wang, E. Furman, and Q. M. Zhang, Science 321, 821 (2008).

${ }^{3}$ J. F. Scott, Annu. Rev. Mater. Res. 41, 229 (2011).

${ }^{4}$ S. Pamir Alpay, J. Mantese, S. Trolier-McKinstry, Q. M. Zhang, and R. W. Whatmore, MRS Bull. 39, 1099 (2014).

${ }^{5}$ Y. Liu, J. F. Scott, and B. Dkhil, APL Mater. 4, 064109 (2016).

${ }^{6}$ Y. Liu, J. F. Scott, and B. Dkhil, Appl. Phys. Rev. 3, 031102 (2016).

${ }^{7}$ R. I. Epstein and K. J. Malloy, J. Appl. Phys. 106, 064509 (2009).

${ }^{8}$ Y. B. Jia and Y. S. Ju, Appl. Phys. Lett. 100, 242901 (2012).

${ }^{9}$ H. Gu, X. S. Qian, X. Li, B. Craven, W. Zhu, A. Cheng, S. C. Yao, and Q. M. Zhang, Appl. Phys. Lett. 102, 122904 (2013).

${ }^{10}$ H. Gu, X. S. Qian, H. J. Ye, and Q. M. Zhang, Appl. Phys. Lett. 105, 162905 (2014).

${ }^{11}$ Y. D. Wang, S. J. Smullin, M. J. Sheridan, Q. Wang, C. Eldershaw, and D. E. Schwartz, Appl. Phys. Lett. 107, 134103 (2015).

${ }^{12}$ W. N. Lawless and C. F. Clark, Phys. Rev. B 36, 459 (1987).

${ }^{13}$ L. Shebanovs, K. Borman, W. N. Lawless, and A. Kalvane, Ferroelectrics 273, 137 (2002).

${ }^{14}$ S. Kar-Narayan and N. D. Mathur, Appl. Phys. Lett. 95, 242903 (2009).

${ }^{15}$ S. Kar-Narayana and N. D. Mathur, J. Phys. D: Appl. Phys. 43, 032002 (2010).

${ }^{16}$ S. Crossley, J. R. McGinnigle, S. Kar-Narayan, and N. D. Mathur, Appl. Phys. Lett. 104, 082909 (2014).

${ }^{17}$ C. Zimm, A. Jastrab, A. Sternberg, V. Pecharsky, K. Gschneidner, Jr., M. Osborne, and I. Anderson, Adv. Cryog. Eng. 43, 1759 (1998).

${ }^{18}$ Y. Bai, G. P. Zheng, and S. Q. Shi, Appl. Phys. Lett. 96, 192902 (2010).

${ }^{19}$ B. Lu, X. H. Wen, Z. H. Tang, B. Liang, T. Tao, Z. W. Xie, T. F. Zhang, X. G. Tang, Y. Xiang, J. Liao, and S. G. Lu, Sci. China: Technol. Sci. 59, 1054 (2016).

${ }^{20}$ Y. Liu, B. Dkhil, and E. Defay, ACS Energy Lett. 1, 521 (2016).

${ }^{21}$ S. Kar-Narayan, S. Crossley, X. Moya, V. Kovacova, J. Abergel, A. Bontempi, N. Baier, E. Defay, and N. D. Mathur, Appl. Phys. Lett. 102, 032903 (2013).
} 